\title{
Identification of m6A-Associated Gene DST as a Prognostic and Immune-Associated Biomarker in Breast Cancer Patients
}

\author{
Xiangyuan Qiu (iD \\ Xinying Li (D) \\ Yuanliang Yan ${ }^{2}$ \\ Yuan $\mathrm{Cai}^{3}$ \\ Qiuju Liang ${ }^{2}$ \\ Bi Peng ${ }^{3}$ \\ Zhijie Xu $\mathbb{1}^{3-5}$ \\ Muzhang $\mathrm{Xiao}^{6}$ \\ Fada Xia' \\ Jinwu Peng ${ }^{3,4}$
}

'Department of Thyroid Surgery, Xiangya

Hospital, Central South University,

Changsha, 410008, Hunan, People's

Republic of China; ${ }^{2}$ Department of

Pharmacy, Xiangya Hospital, Central South

University, Changsha, 410008, Hunan,

People's Republic of China; ${ }^{3}$ Department of Pathology, Xiangya Hospital, Central South University, Changsha, 410008, Hunan, People's Republic of China; ${ }^{4}$ Department of Pathology, Xiangya Changde Hospital, Changde, 4I5000, Hunan, People's

Republic of China; ${ }^{5}$ National Clinical Research Center for Geriatric Disorders, Xiangya Hospital, Central South University, Changsha, 4I0008, Hunan, People's Republic of China; ${ }^{6}$ Department of Burn and Plastic Surgery, Xiangya Hospital, Central South University, Changsha, 410008, Hunan, People's Republic of China

Correspondence: Muzhang Xiao Department of Burn and Plastic Surgery, Xiangya Hospital, Central South University, Changsha, 410008, Hunan, People's Republic of China

Email xmzsir@sina.com

Fada Xia

Department of Thyroid Surgery, Xiangya

Hospital, Central South University,

Changsha, 4I0008, Hunan, People's

Republic of China

Email xiafada@csu.edu.cn
Background: N6-methyladenosine (m6A) is most common internal RNA modification in eukaryotic cells. Existing evidence shows that $\mathrm{m} 6 \mathrm{~A}$ is closely related to pathogenesis and progression in breast cancer (BRCA). Therefore, it is critical to investigate the key role of m6A target genes in BRCA.

Methods: M6A target genes in BRCA are acquired using RMVar online database. The differentially expressed genes (DEGs) from three microarray datasets (GSE5764, GSE22358, GSE9014) is processed by GEO2R. Oncomine, GEPIA, UALCAN and TNMplot were applied to validate the expression of DST. Survival analyses were performed via DRUGSURV and Kaplan-Meier Plotter database. Univariable survival and multivariate Cox analysis were completed to assess the prognostic value of DST and receiver operating characteristic (ROC) curve was performed to evaluate the diagnostic value of DST. We also investigated the correlation between DST and cancer immune infiltration via using CIBERSORT, TIMER and TISIDB.

Results: DST and COL11A1 were significantly expressed in both DEGs and m6A target genes set. COL11A1 show no significance on the patients' survival. However, high expression of DST was related to the favorable prognosis. Multivariate analysis revealed that the DST dysregulation is an independent prognostic factor and ROC indicated that the great diagnostic value of DST with AUC of 0.948. Subsequently, immunological analyses showed that DST was significantly associated with various immune infiltration cells, including NK cells, T helper cells and Mast cells. Furthermore, DST was also related with multiple immune checkpoints and chemokines, including LAG3, LMTK3 CD24, CXCL12, KDR and CX3CR1. These results indicated the potential roles of DST in the development of BRCA via altering the immune response.

Conclusion: DST can influence the development and progression of BRCA by altering the immune microenvironment.

Keywords: breast cancer, DST, N6-methyladenosine, immune microenvironment, immune infiltration

\section{Introduction}

In women, breast cancer (BRCA) is the most common cancer and the first cause of cancer death surpassing lung cancer. BRCA is a heterogeneous disease composed of various biological subtypes. ${ }^{1,2}$ The administration of therapeutic targets based on molecular mechanism can improve clinical outcomes such as hormonal therapy and HER2-targeted therapy. ${ }^{3}$ Therefore, the discovery of novel therapeutic targets is critical for BRCA patients. 
N6-methyladenosine (m6A) is a dynamic modification and its removal and installation have been demonstrated to be an important component of gene expression alteration. ${ }^{4,5}$ M6A can regulate RNA function and metabolism ranging from RNA splicing, nuclear export and translation to RNA degradation. ${ }^{6,7}$ The occurrence and disappearance of m6A is distributed to three regulators: "writers"; "erasers"; "readers". To decide the fate of modified RNA, the "writers" generate m6A mark, "erasers" show demethylation activity and "readers" recognize m6A modification. ${ }^{8}$ Accumulating evidence indicates that $\mathrm{m} 6 \mathrm{~A}$ methylation plays a significant role in cancer pathogenesis via different mechanisms. YTHDF1 increases the translation of EIF3C via binding to m6Amodified EIF3C mRNA and promotes the tumorigenesis and metastasis of ovarian cancer. ${ }^{9}$ METTL 3 interacts with the microprocessor protein DGCR8 and positively regulates the pri-miR221/222 process in an m6A-dependent manner, which plays an oncogenic role in bladder cancer. ${ }^{10}$ IGF2BP can augment the expression of SRF in m6A-dependent manner by blocking the miRNA-directed decay of the SRF mRNA, which promotes tumor cell invasion and growth. ${ }^{11}$ Thus, understanding the detailed roles of m6A in BRCA may provide us with more possibilities for treatments.

Cancer cells can functionally remodel tumor microenvironment (TME) via secreting diverse cytokines and chemokines. ${ }^{12}$ Immune cells are important constituents of the tumor stroma. ${ }^{13}$ Immune cells are composed of innate immune cells (neutrophils, macrophages, innate lymphoid cells, natural killer cells, dendritic cells, suppressor cells and myeloid-derived) and adaptive immune cells ( $\mathrm{T}$ cells and B cells). Immune cells within TME play tumorpromoting and tumor-antagonizing properties of tumors. ${ }^{14}$ Accordingly, immunotherapy has become a promising field and therapeutic strategy. ${ }^{15-17}$

In this study, we identified a differentially expressed m6A target gene, DST, and analyzed the clinical significance of DST. Furthermore, the immunoregulatory role of DST was also explored in BRCA patients.

\section{Methods}

\section{Data Acquisition}

Three microarray datasets related with BRCA (GSE5764, GSE22358, GSE9014) were download from Gene Expression omnibus (GEO) database. The differentially expressed genes is obtained by GEO2R tool ${ }^{18}$ (https:// www.ncbi.nlm.nih.gov/geo/geo2r/). The adj. P value $<0.05$ and $|\operatorname{logFC}|>1$ were set as a cutoff criterion. M6A target genes in BRCA are acquired by RMVar online database ${ }^{19}$ (http://www.rmvar.renlab.org/browse.html). The co-DEGs among three dataset and m6A target genes were intersected by Venn diagram. Gene expression and clinical data were downloaded from the Cancer Genome Atlas (TCGA) website (https://portal.gdc.cancer.gov/). Clinical profiles, including age, sex, $\mathrm{T}$ stage, $\mathrm{N}$ stage, $\mathrm{M}$ stage, and follow-up data, were collected. Patients with incomplete date were deleted. Finally, a total of 1069 patients were included.

\section{Expression and Clinical Significance Analysis}

Gene expression was obtained from Oncomine ${ }^{20}$ (http:// www.oncomine.org) and validated by GEPIA ${ }^{21}$ (http:// gepia.cancerpku.cn/), UALCAN ${ }^{22}$ (http://ualcan.path.uab. edu/analysis.html), and TNMplot (https://www.tnmplot. com/). ${ }^{23}$ With the help of Kaplan-Meier Plotter ${ }^{24}$ (http:// kmplot.com/analysis/) and DRUGSURV ${ }^{25}$ (http://www.bio profiling.de/GEO/DRUGSURV/index.html), survival analysis was accomplished. The diagnostic value is assessed by the receiver operating characteristic (ROC) curve. Univariate and multivariate Cox regression analysis were performed to evaluate the prognostic power.

\section{Correlation and Functional Enrichment Analysis}

The co-expressed genes were acquired from LinkedOmics database $^{26}$ (http://www.linkedomics.org/login.php). Then, Gene Ontology biological process (GO_BP), Gene Ontology cellular component (GO_CC), Gene Ontology molecular function (GO_MF) and Kyoto Encyclopedia of Genes and Genomes (KEGG) pathways were analyzed by the gene set enrichment analysis (GSEA) in the LinkInterpreter module.

\section{Immunological Analysis}

The correlations between gene expression and tumourinfiltrating immune cells from TCGA were performed. Tumor Immune Estimation Resource (TIMER) database ${ }^{27}$ (https://cistrome.shinyapps.io/timer/) and TISIDB database ${ }^{28}$ (http://cis.hku.hk/TISIDB) were applied for validation. Simultaneously, immune score of every patient is generated with ESTIMATE algorithm.

\section{Statistical Analyses}

R 4.1.0 and Statistical Package for Social Sciences 26.0 for Windows (SPSS Inc, Chicago, IL, United States) were used to perform statistical analyses. Clinicopathological 
parameters were compared using chi-square or Fisher's exact tests.

Univariate and multivariate analyses were conducted using the Cox-regression method. The pROC package ${ }^{29}$ was applied for ROC analysis. $\mathrm{P}$ value $<0.05$ was significant difference. The main tools involved in this study are summarized in Table 1.

\section{Results}

\section{Identification of m6A Target Genes}

The BRCA datasets of GSE5764, GSE22358 and GSE9014 were acquired to identify differentially expressed genes by GEO2R. 9308 DEGs was found in GSE9014, while 1538 DEGs in GSE22358 and 45 DEGs in GSE8977. As shown in Figure 1A, Venn plot showed 11 co-DEGS. We download m6A target geneset in BRCA from RMVar online database. Venn plot (Figure 1B) between co-DEGS and m6A target geneset depicted two m6A target genes (DST and COL11A1). Next, the prognostic values of DST and COL11A1 were explored by using survival data of BRCA patients from DRUGSURV databases. Survival curves of DST (Figure 1C and D) are statistically significant in both GSE11121 and GSE17705, but COL11A1 (Figure 1E and F) is not. Therefore, further investigations were focused on DST.

\section{Validation of the Expression Level and Clinical Significance of DST}

As depicted in Figure 2A, the expression of DST from Oncomine database was downregulated in various cancers, including BRCA. Subsequently, three online tools, UALCAN, TNMplot and GEPIA, were used to validate the downregulated DST (Figure 2B-D). Patients were divided into two groups according to the expression of DST. The association of DST expression with clinicopathological parameters is shown in Table 2. There was no significant difference in $\mathrm{T}, \mathrm{N}, \mathrm{M}$ stage and sex between the two groups. High expression of DST was significantly associated with the older age $(p=0.002)$. Moreover, in TCGA-BRCA, the patients with low level of DST displayed poor survival probability $(\mathrm{p}=0.0054)$ and the hazard ratio (HR) is 0.63 (95\% confidence interval [CI], 0.46-0.88) (Figure 2E). Univariate and multivariate Cox regression analyses (Table 3 ) indicate that DST is an independent prognostic factor (Figure 2F). The ROC curve showed a great diagnostic value of DST with AUC of 0.948 (95\% CI: 0.925-0.970) (Figure 2G).

\section{DST Co-Expression and Enrichment Analyses}

To explore the biological function of DST in BRCA, the LinkedOmics tool was used to obtain the co-expression pattern of DST in TCGA-BRCA. 5785 genes showed a negative correlation with DST, while 9003 genes showed a positive correlation (Figure 3A). Figure $3 \mathrm{~B}$ and $\mathrm{C}$ showed the top 50 genes positively and negatively related to DST, respectively. Enrichment analysis was completed to explore the potential biological functions of DST. The enrichment results of GO terms and KEGG pathway are shown in Figure 3D-G. Mitochondrial respiratory chain complex assembly, mitochondrial protein complex and structural constituent of ribosome were the top enriched terms in biological process, cellular component, and molecular functions, respectively. Proteasome, ribosome and oxidative phosphorylation were the top three enriched KEGG pathways.

Table I The Main Tools Involved in This Study

\begin{tabular}{|c|c|c|c|}
\hline Database & Samples & URL & References \\
\hline GEO2R & Tissue & https://www.ncbi.nlm.nih.gov/geo/geo2r/ & {$[18]$} \\
\hline RMVar & Tissue & http://www.rmvar.renlab.org/browse.html & {$[19]$} \\
\hline Oncomine & Tissue & http://www.oncomine.org & [20] \\
\hline GEPIA & Tissue & http://gepia.cancerpku.cn/ & {$[21]$} \\
\hline UALCAN & Tissue & http://ualcan.path.uab.edu/analysis.html & [22] \\
\hline TNMplot & Tissue & https://www.tnmplot.com/ & [23] \\
\hline Kaplan-Meier Plotter & Tissue & http://kmplot.com/analysis/ & [24] \\
\hline DRUGSURV & Tissue & http://www.bioprofiling.de/GEO/DRUGSURV/index.html & {$[25]$} \\
\hline LinkedOmics & Tissue & http://www.linkedomics.org/login.php & [26] \\
\hline TIMER & Tissue & https://cistrome.shinyapps.io/timer/ & [27] \\
\hline TISIDB & Tissue & http://cis.hku.hk/TISIDB & [28] \\
\hline
\end{tabular}


A

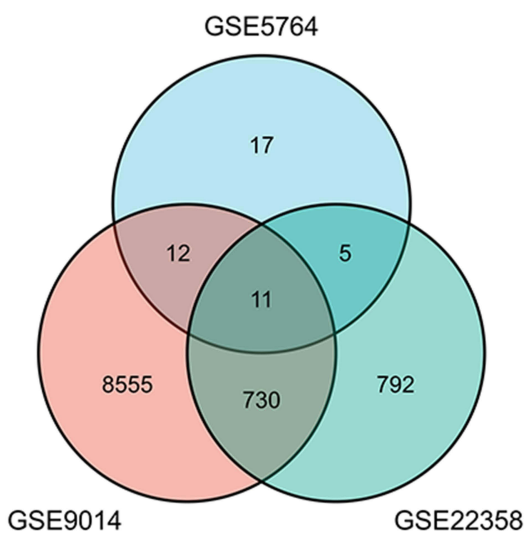

C

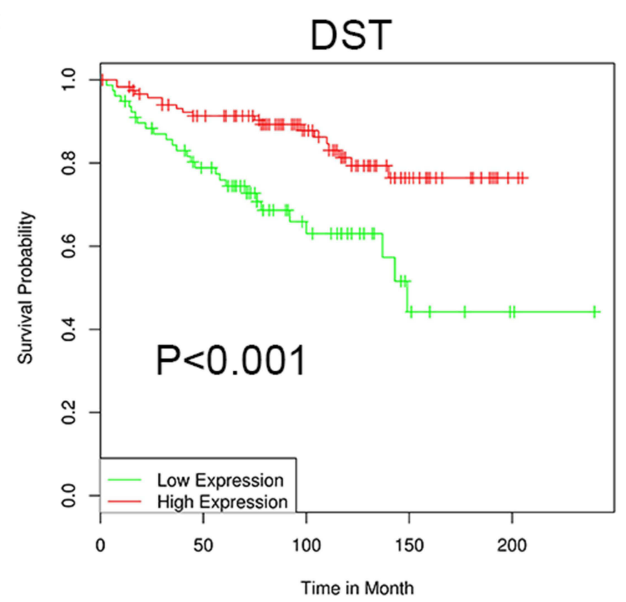

GSE11121

E

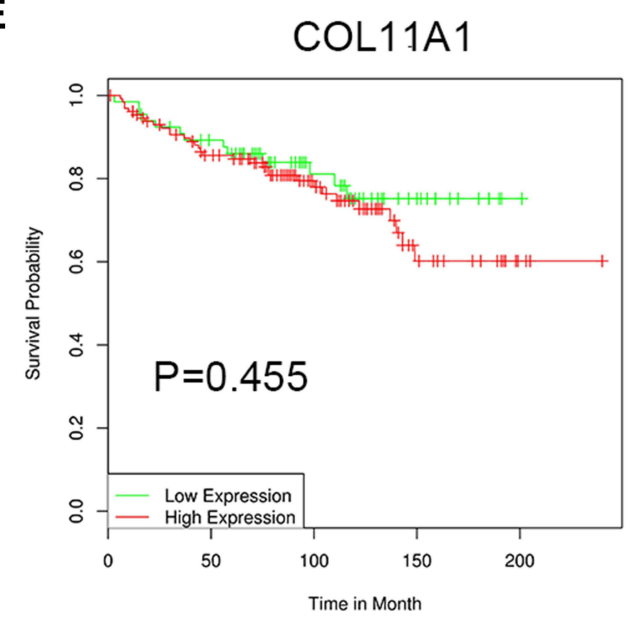

GSE11121
B

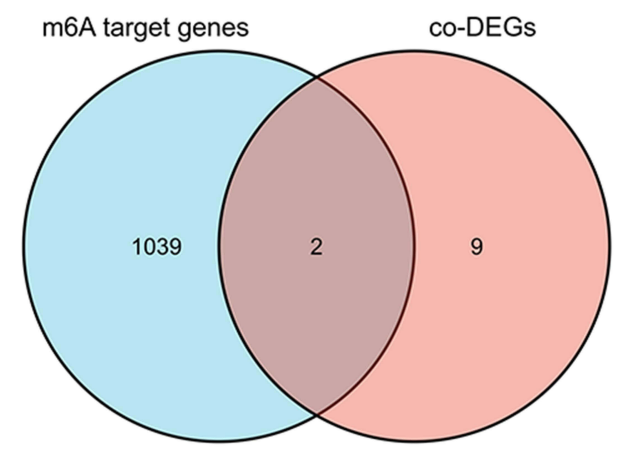

D

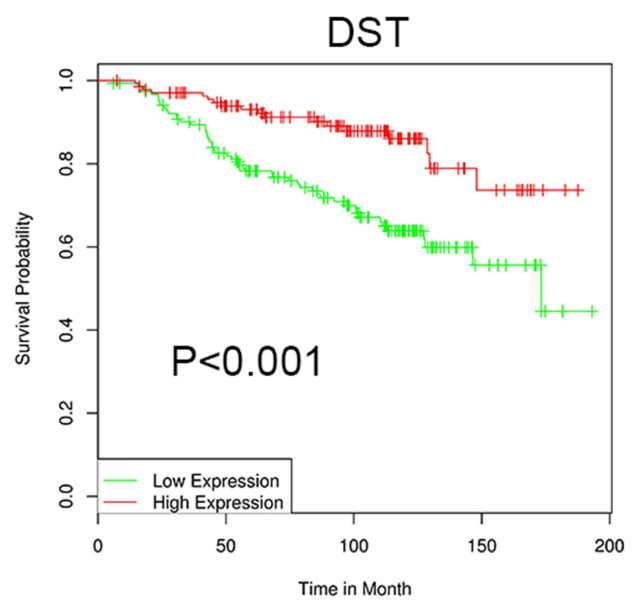

GSE17705

F

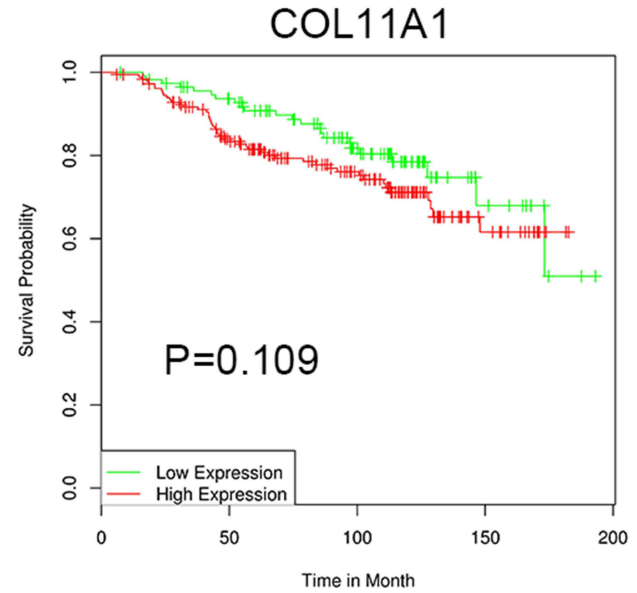

GSE17705

Figure I Identification of m6A target genes. (A) Venn plots of co-DEGs. (B) Venn plots of m6A target co-DEGs. (C-F) Kaplan-Meier curves of DST (C and D) and COLIIAI (E and F) in GSEIIIII and GSEI7705. 
A

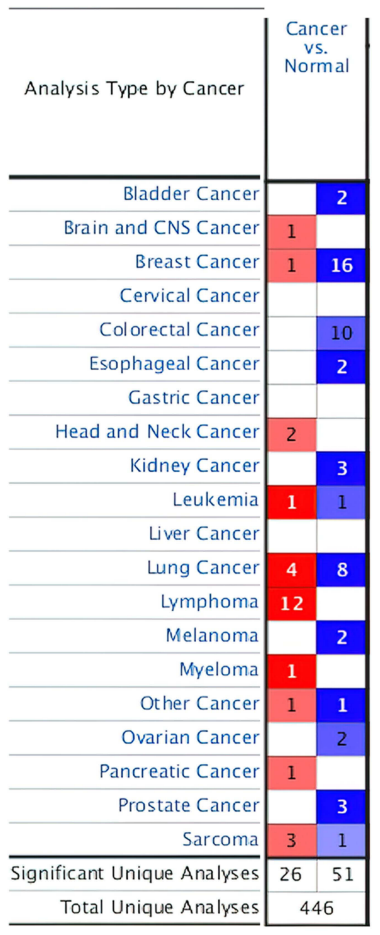

B

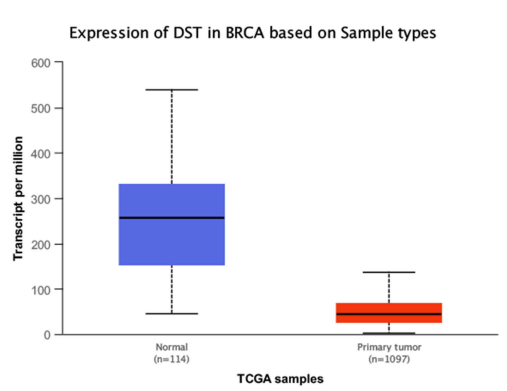

D

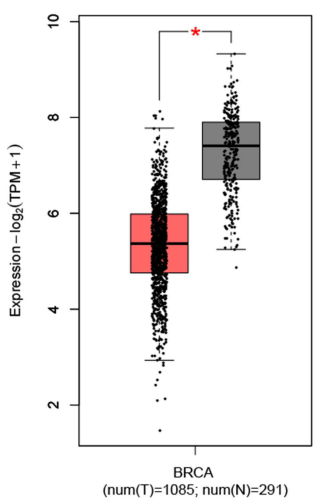

C

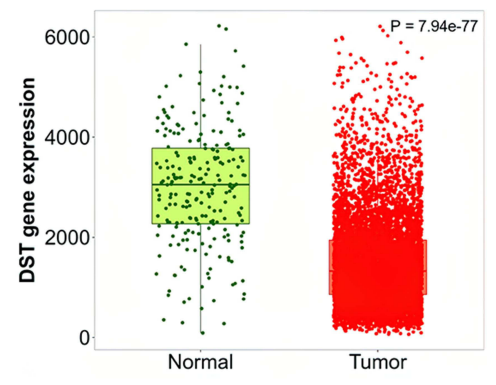

E

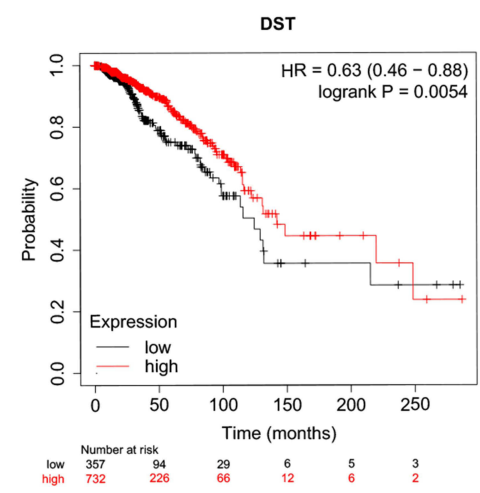

G

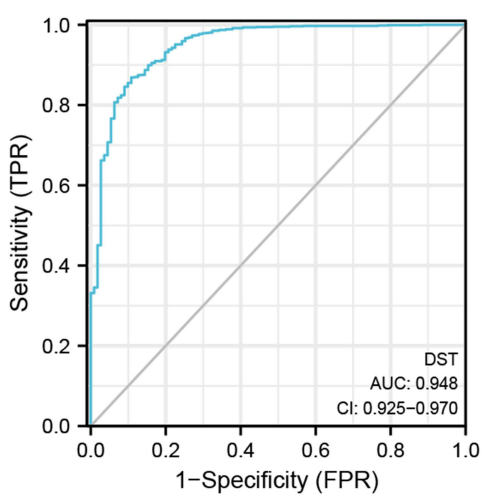

Figure 2 Validation of the expression and clinical significance of DST. (A-D) The expression of DST in breast cancer and normal validated by oncomine (A), UCLCAN (B), TNM plot (C), GEPIA (D). (E) The Kaplan-Meier curve of DST on overall survival of TCGA-BRCA. (F) Forest plot of risk factor. (G) The ROC curve of DST on diagnosing breast cancer. $* \mathrm{P}<0.05$.

\section{The Role of DST in the Immune Microenvironment}

The TME is increasingly recognized as an important factor in the development and progression of BRCA. ${ }^{30}$ Immune score of TCGA-BRCA, calculated using the ESTIMATE package, ${ }^{31}$ showed that patients with higher immune score had a better OS (Figure 4A). To investigate the role of DST in the immune microenvironment of BRCA, we further explored associations between DST expression and immune infiltration cells using ssGSEA algorithm. NK cells, T helper cells, Mast cells, eosinophils, Tgd, Dendritic cells (DC), macrophages, B cells, TFH and neutrophils were found to be positively correlated with DST in BRCA (Figure 4B). The top three cell types (NK cells, Mast cells, T helper cells) were validated using the TISIDB database (Figure 4C-E) and TIMER (Figure 4F-H) database, indicating that DST might influence the immune microenvironment in BRCA patients. Additionally, we also observed the negative associations 
Table 2 The Characteristics of BRCA Patients from TCGA Database

\begin{tabular}{|c|c|c|c|}
\hline Characteristic & $\begin{array}{l}\text { Low Expression of DST } \\
\qquad(n=309)\end{array}$ & $\begin{array}{l}\text { High Expression of DST } \\
\qquad(n=760)\end{array}$ & $P$ value \\
\hline \multicolumn{4}{|l|}{ Age } \\
\hline$\leq 60$ & 150 & 448 & 0.002 \\
\hline$>60$ & 159 & 312 & \\
\hline \multicolumn{4}{|l|}{ Gender } \\
\hline Female & 302 & 755 & 0.052 \\
\hline Male & 7 & 5 & \\
\hline \multicolumn{4}{|l|}{ T stage } \\
\hline$T_{1}-T_{2}$ & 257 & 639 & 0.616 \\
\hline $\mathrm{T}_{3}-\mathrm{T}_{4}$ & 52 & 118 & \\
\hline \multicolumn{4}{|l|}{ M stage } \\
\hline$M_{0}$ & 247 & 643 & 0.6743 \\
\hline$M_{1}$ & 7 & 15 & \\
\hline \multicolumn{4}{|l|}{$\mathrm{N}$ stage } \\
\hline $\mathrm{N}_{0}-\mathrm{N}_{\mathrm{l}}$ & 244 & 615 & 0.458 \\
\hline $\mathrm{N}_{2}-\mathrm{N}_{3}$ & 60 & 133 & \\
\hline \multicolumn{4}{|l|}{ AJCC stage } \\
\hline Stagel-II & 215 & 530 & 0.560 \\
\hline StagellI-IV & 80 & 180 & \\
\hline
\end{tabular}

Table 3 Univariate and Multivariate Analysis with OS in BRCA Patients

\begin{tabular}{|c|c|c|c|c|c|c|}
\hline \multirow[t]{2}{*}{ Clinicopathologic Parameter } & \multicolumn{3}{|c|}{ Univariate Analysis } & \multicolumn{3}{|c|}{ Multivariate Analysis } \\
\hline & HR & $95 \% \mathrm{Cl}$ & $P$ value & HR & $95 \% \mathrm{Cl}$ & $P$ value \\
\hline Age (>60 vs $\leq 60)$ & 1.97 & $1.42-2.7$ & $<0.001$ & 2.00 & $|.38-2.9|$ & $<0.001$ \\
\hline Gender (male vs female) & 0.83 & $0.12-5.95$ & 0.854 & 0.53 & $0.07-3.87$ & 0.532 \\
\hline T stage (T3-T4 vs TI-T2) & 1.80 & $1.25-2.59$ & 0.002 & 0.96 & $0.56-1.62$ & 0.864 \\
\hline M stage (MI vs M0) & 4.90 & $2.93-8.21$ & $<0.001$ & 2.27 & I. $17-4.40$ & 0.016 \\
\hline $\mathrm{N}$ stage $(\mathrm{nl}$-n2 vs n0-nI) & 2.30 & I.58-3.37 & $<0.001$ & 0.97 & $0.53-1.76$ & 0.911 \\
\hline AJCC Stage (III-IVvsI-II) & 2.67 & $1.92-3.72$ & $<0.001$ & 2.63 & I.36-5.07 & 0.004 \\
\hline DST expression (high vs low) & 0.65 & $0.47-0.91$ & 0.12 & 0.68 & $0.46-0.99$ & 0.045 \\
\hline
\end{tabular}

Abbreviations: HR, hazard ratio; $\mathrm{Cl}$, confidence interval; OS, overall survival; AJCC, American Joint Committee on Cancer.

between DST levels and multiple immune checkpoint molecules, including LAG3 (Figure 4I), LMTK3 (Figure 4J) and CD24 (Figure 4K).

Further investigations were focused on extending the recognition of immune roles of DST. TISIDB database was applied to analyze the associations between DST with immunostimulators, immunoinhibitors, chemokines, and receptors. As shown in (Figure 5A and B), multiple immunostimulators and immunoinhibitors showed associations with the levels of DST, with CXCL12 (Spearman: $\rho=0.312$ ) as the most significant immunostimulatory marker and KDR (Spearman: $\rho=0.39$ ) as the most significant immunoinhibitory marker. Simultaneously, Figure $6 \mathrm{~A}$ and B showed that CXCL12 (Spearman: $\rho=0.312$ ) was the most significant chemokine and CX3CR1 (Spearman: $\rho=0.344$ ) was the most significant chemokine receptors. Next, we wanted to verify the associations between these molecules in above-mentioned GEO datasets. As shown in Table S1, CXCL12 was still significantly related with DST in GSE22538 (Spearman: $\rho=4.94 \mathrm{E}-13$ ) and GSE9014 (Spearman: $\rho=0.038$ ). Interestingly, KDR was also significantly related with DST in GSE22538 (Spearman: $\rho=1.93 \mathrm{E}-9$ ) and GSE9014 (Spearman: $\rho=3.72 \mathrm{E}-3$ ). However, no significant association could be found in GSE5764, which needs to be further clarified. These results together suggested that DST might play a critical role in the progression and development of patients with BRCA by regulating the immune response. 


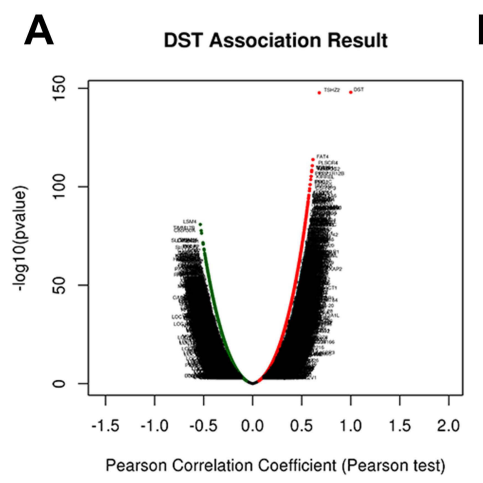

D

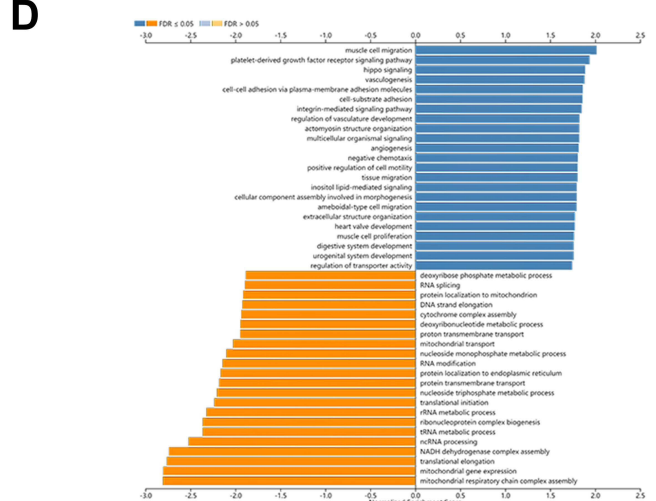

F

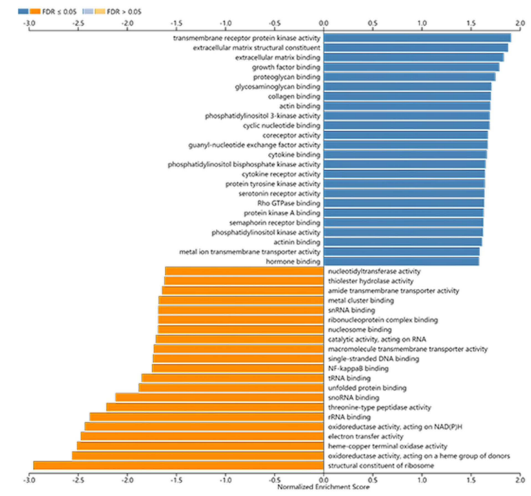

C

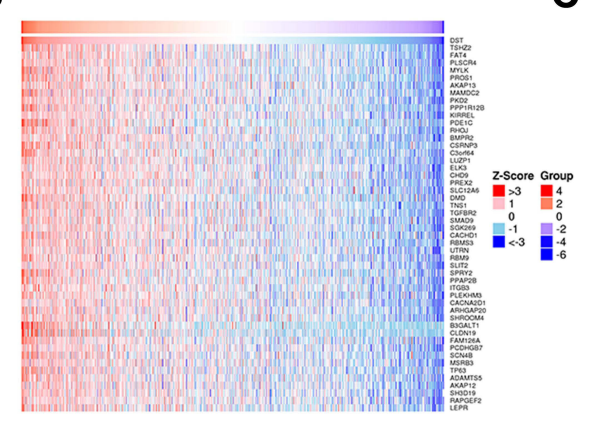

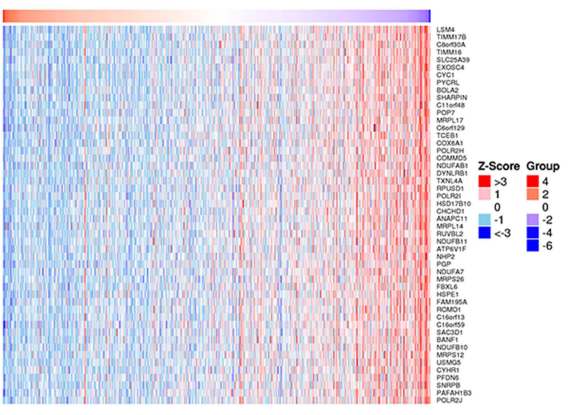

E

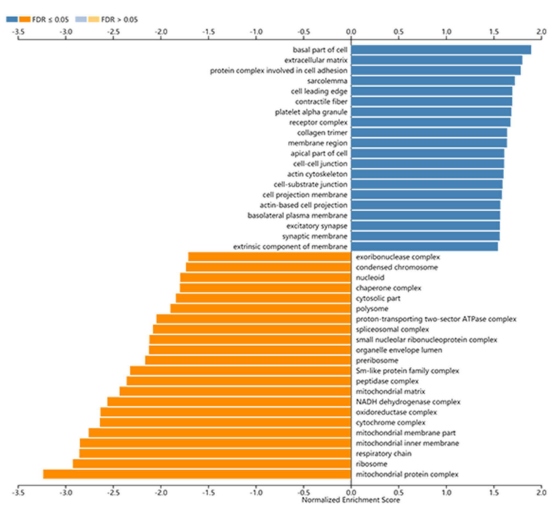

G

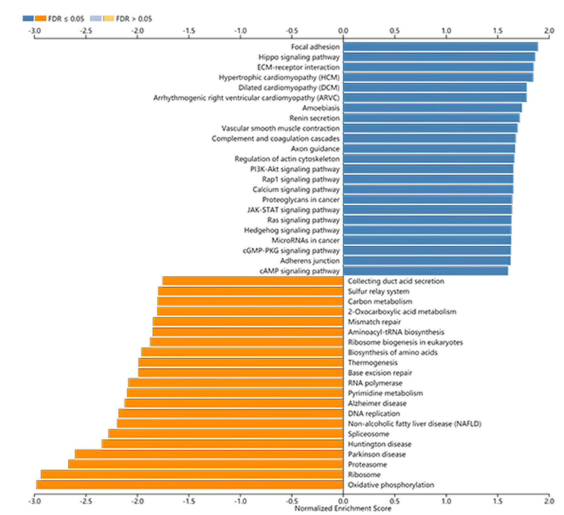

Figure 3 The co-expression genes with DST from the LinkedOmics database. (A) Genes significantly related with DST by Pearson test. (B and C) Heat maps of top 50 genes positively and negatively correlated to DST. Red represents genes positively correlated and blue represents genes negatively associated. (D-G) GO terms for molecular functions (D), biological process (E), cellular component (F) and KEGG pathways (G) of DST by GSEA analyses.

\section{Discussion}

In this study, we found a m6A target gene, DST, plays an important role in the pathogenesis and development of BRCA, acting as a potential tumor suppressor. Further investigations showed that the DST was involved with the immune microenvironment of BRCA, indicating that DST might make an impact on BRCA by affecting the immune microenvironment. These results suggested that DST might be reckoned as a biomarker or therapeutic target in patients with BRCA.
There are several studies introducing the importance of m6A in BRCA. M6A is the most abundant internal modification of RNA and is enriched in various cancers PMID: 32355831. M6A modification is installed by the m6A methyltransferases, removed by the demethylases and are recognized by m6A-binding proteins, which alter gene expression via regulating RNA metabolism, including translation, splicing, export and degradation. ${ }^{32} \mathrm{M} 6 \mathrm{~A}$ target genes alteration in BRCA can modulate cellular processes including cell growth, metastasis, invasion and apoptosis. ${ }^{33-35}$ 
A

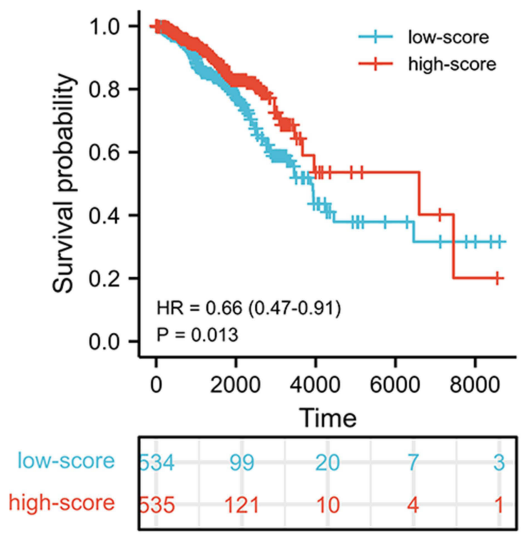

B

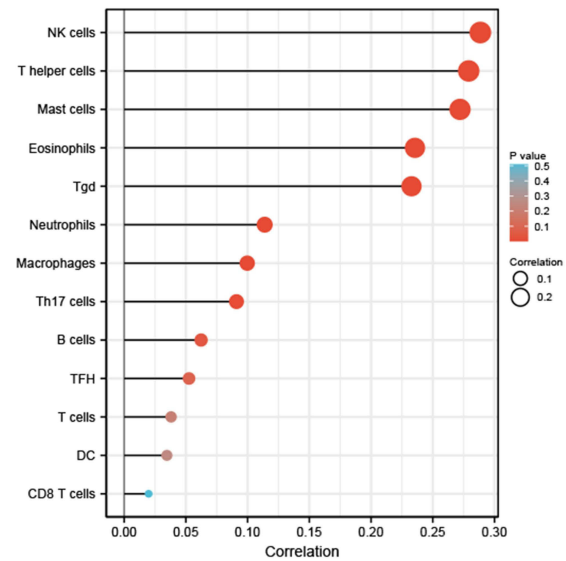

F

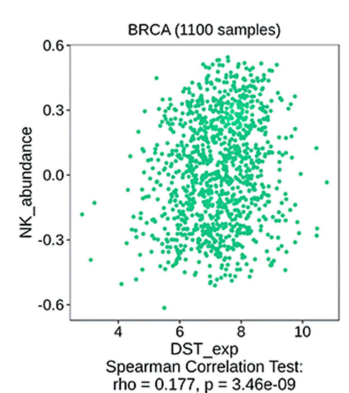

$\mathbf{G}$

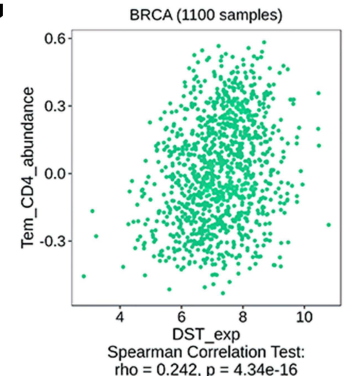

H

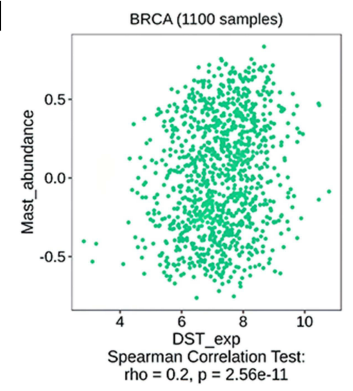

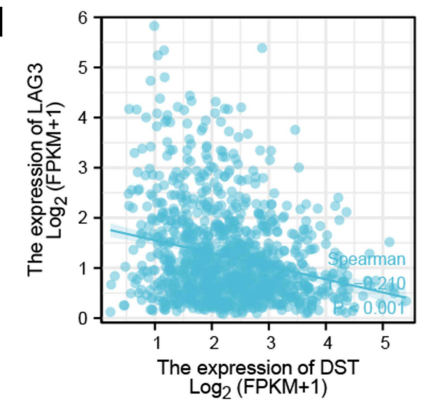

J

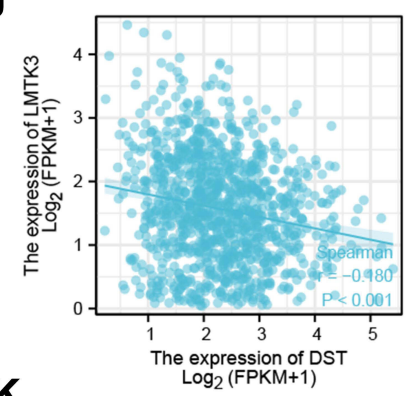

K

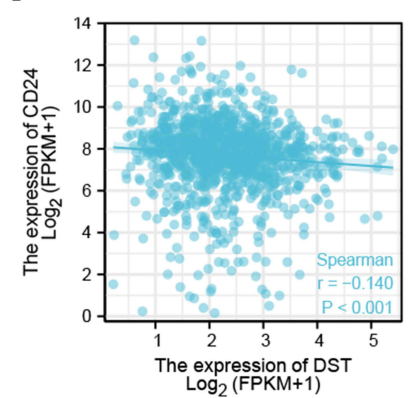

Figure 4 The associations between immune infiltration and DST. (A) The Kaplan-Meier curve of immune score on overall survival of TCGA-BRCA. (B) Immune infiltration cells associated with DST using ssGESA algorithm. Validations of top three cells by TIMER database. (C-E) Validations of top three cells by TISIDB database, such as NK cells (C), T helper cells (D), Mast cells (E). (F-H) Validations of top three cells by TIMER database, such as NK cells (F), T helper cells (G), Mast cells (H). (I-K) The correlation between DST with LAG3 (I), LMTK3 (J) and CD24 (K). 
A

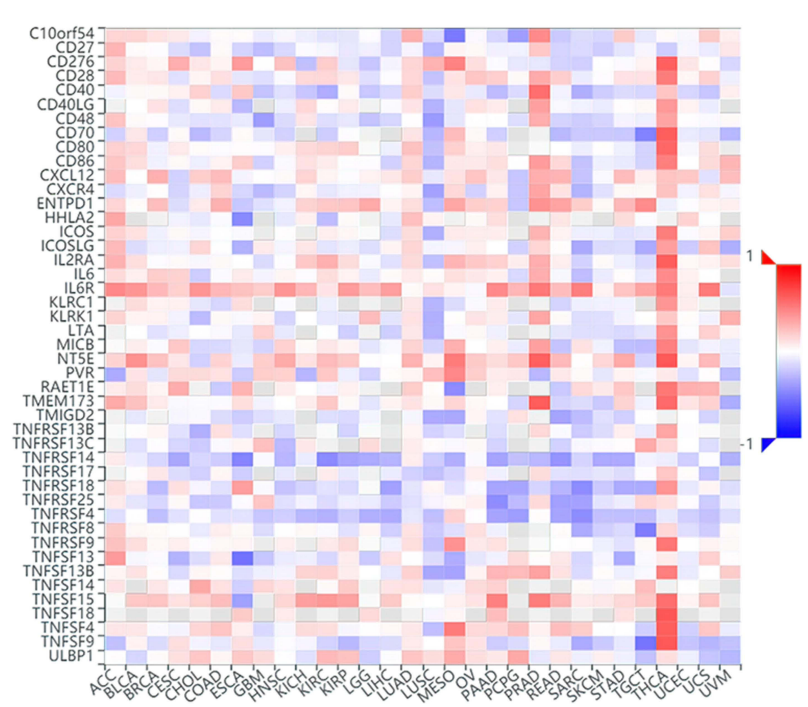

B

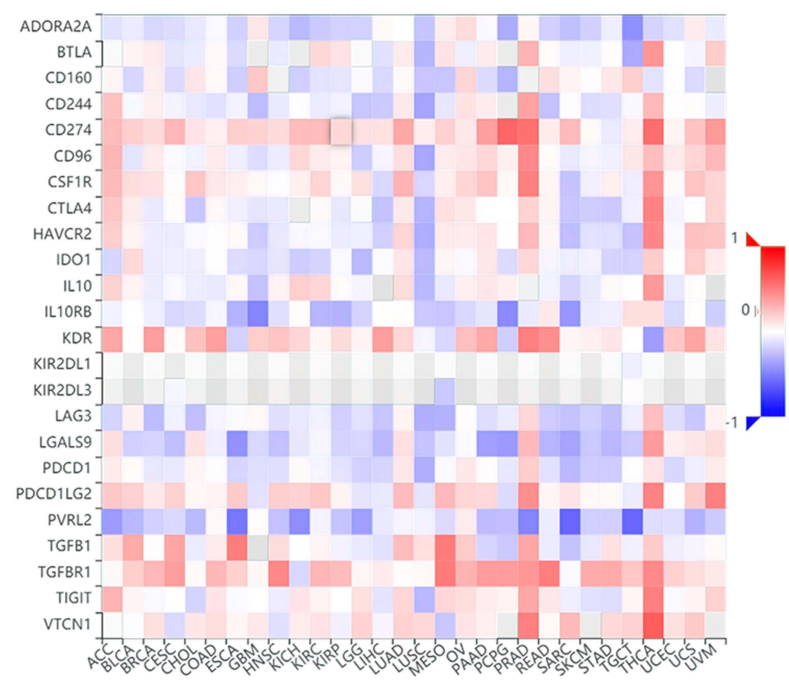

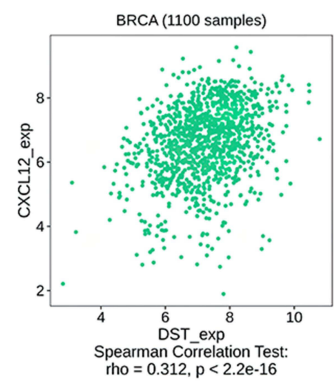
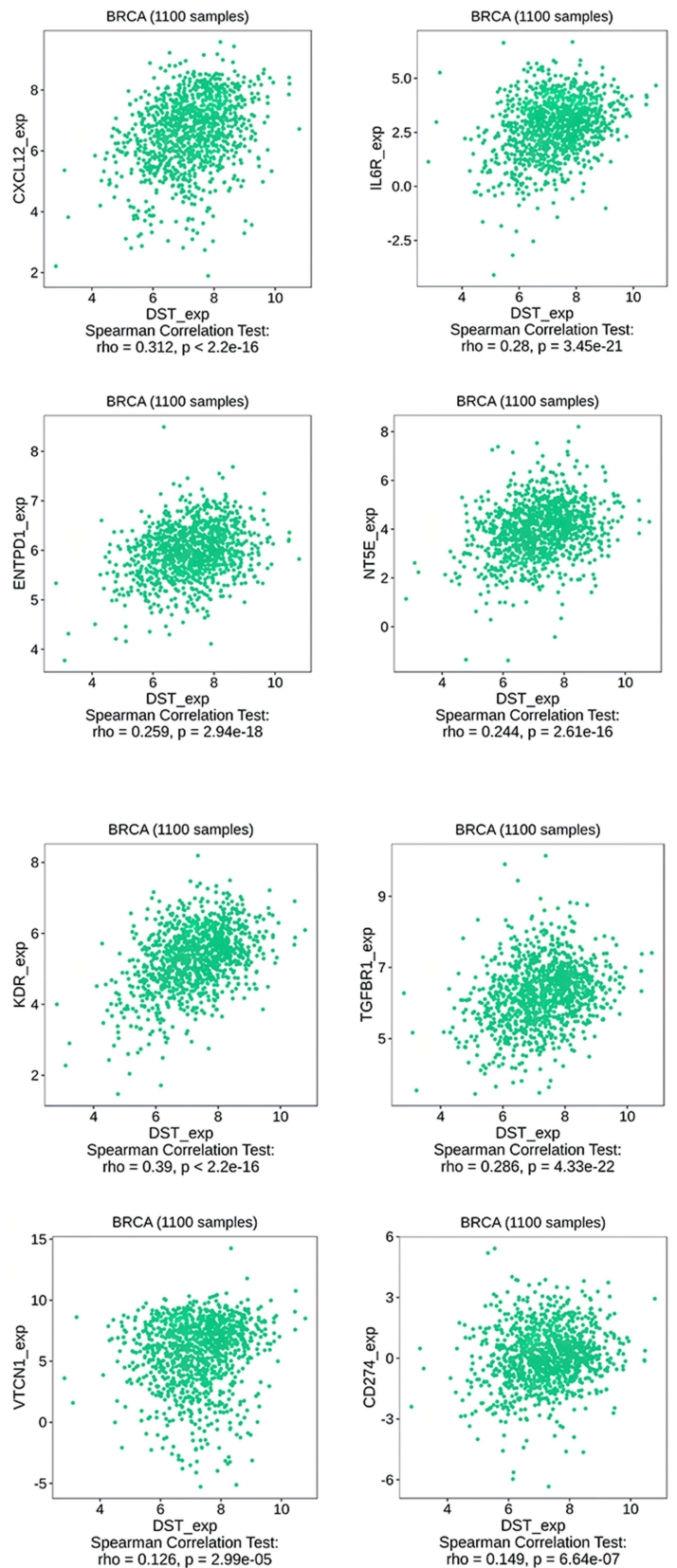

Figure 5 Associations of the DST expression with immunomodulators from TISIDB database. (A) Correlations between immunostimulators and DST with four most significant molecules. (B) Correlations between immunoinhibitors and DST with four most significant molecules.

We obtained a list of m6A target genes from RMVar online database. These genes were intersected with DEGs from three GEO datasets. We acquired DST and COL11A1. According to survival data from DRUGSURV database, DST was selected as a candidate. Subsequently, we validated the levels of DST in BRCA with Oncomine and three online databases. Additionally, we also found that DST was downregulated in many cancers, but little mechanism was explored in human cancer.
We explored the clinical significance of DST. DST was an independent prognostic factor via multivariate Cox regression analyses and the ROC curve showed a great diagnostic value of DST with an AUC of 0.948. Higher expression of DST was accompanied by better survival outcomes, but there was no significant correlation between $\mathrm{T}, \mathrm{N}, \mathrm{M}$ stage and sex.

The above results reveal that DST might be a critical biomarker in BRCA. Recently, increasing evidence has 
A
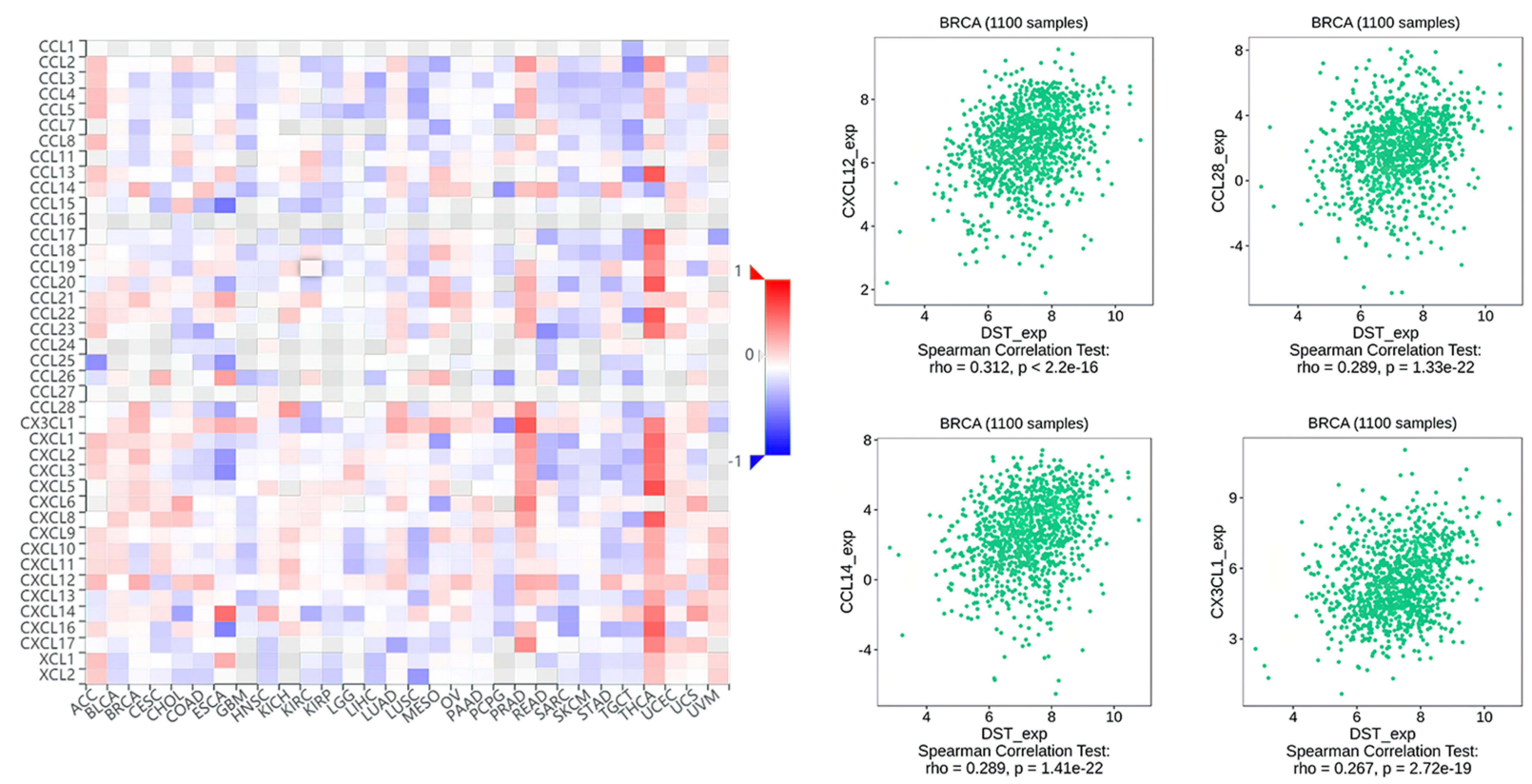

B
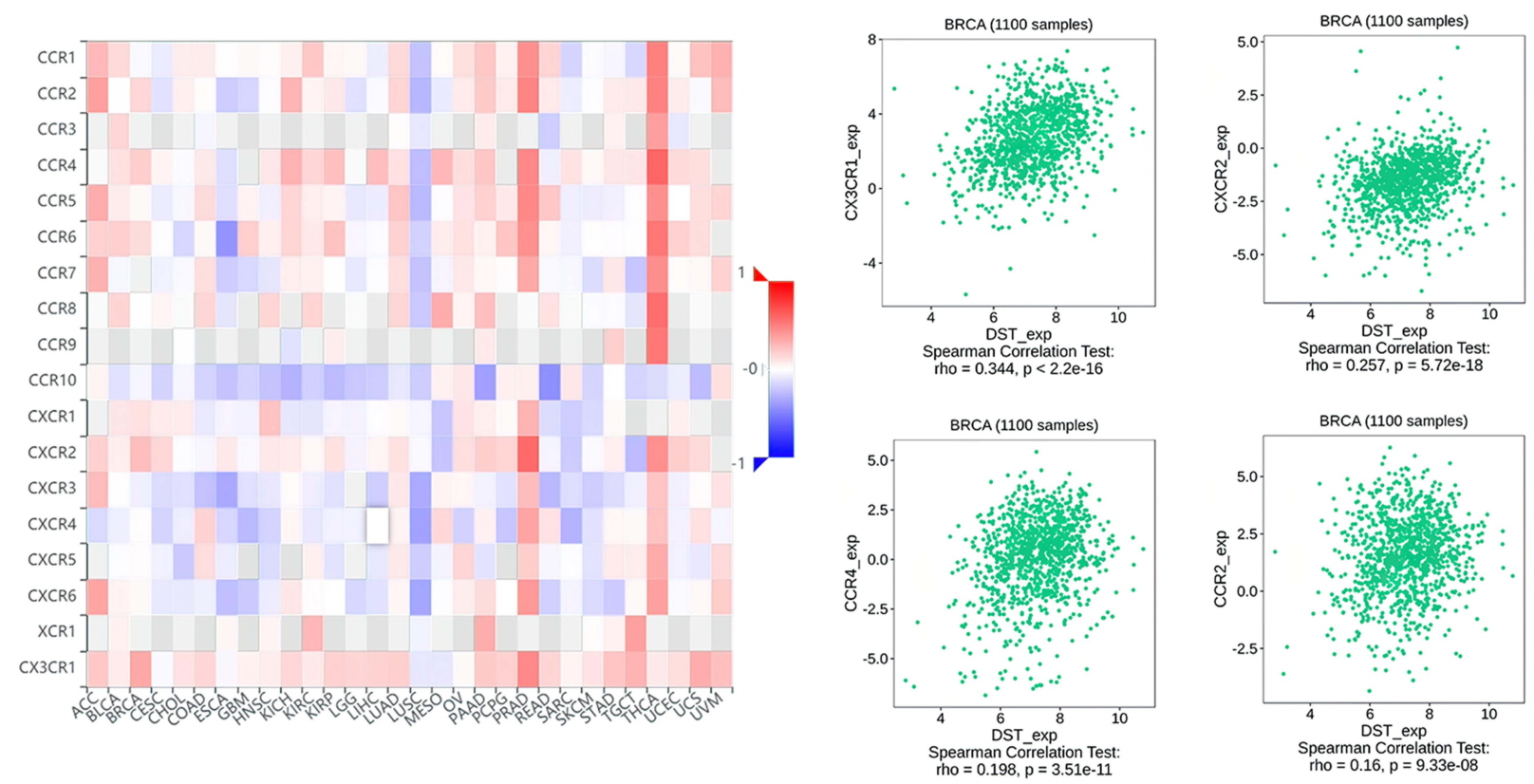

Figure 6 Associations of the DST expression with chemokines and receptors from TISIDB database. (A) Correlations between chemokines and DST with four most significant molecules. (B) Correlations between receptors and DST with four most significant molecules.

shown that the degree of heterogeneity of the TEM in BRCA could be recognized as an important element for cancer development and progression, as well as potential therapeutic targets. ${ }^{36-38}$ Therefore, we investigated the relevance between DST and tumor immune microenvironment. DST was positively correlated with multiple immune cells and negatively correlated with many immune checkpoints. The top three cell types (NK cells, Mast cells, $\mathrm{T}$ helper cells) were validated using the
TISIDB and TIMER databases. NK cells function as the main effector cells toward cancer in innate immunity, ${ }^{39}$ and inhibition of NK cells leads to immune escape and multi-step metastatic process of BRCA. ${ }^{40}$ Mast cells play a dual role in both anti-tumoral and pro-tumoral functions in BRCA, ${ }^{41}$ which highlights the importance of personalized treatments. $\mathrm{T}$ helper cells are critical regulators of immune with great therapeutic and prognostic value in BRCA. ${ }^{42,43}$ In this study, we found DST might function 
via altering the immune microenvironment of BRCA, and this is the first study reporting the correlation between DST and immune microenvironment in BRCA.

There were several limitations in this study. These results were mainly derived from the bioinformatics analysis but lacked adequate experimental validation. More experimental and clinical researches were needed to further validate the biological role of DST in breast cancer.

\section{Conclusion}

In conclusion, DST may alter the development and progression of BRCA via influencing the immune microenvironment and possess diagnostic and therapeutic values to some extent.

\section{Abbreviations}

BRCA, breast cancer; GEO, gene expression omnibus; coDEGs, co-differentially expressed genes; ROC, receiver operating characteristic; GO_BP, Gene Ontology biological process; GO_CC, Gene Ontology cellular component; GO_MF, Gene Ontology molecular function; KEGG, Kyoto Encyclopedia of Genes and Genomes; TIMER, Tumor Immune Estimation Resource; NK, Natural killer cells; TME, tumor microenvironment.

\section{Data Sharing Statement}

All data generated or analyzed during this study are included in this published article.

\section{Ethics Approval}

All our data belong to public databases and online websites, which are based on open source. The patients involved in the database have obtained ethical approval. Users can download relevant data for free for research and publishing relevant articles, so there are no ethical issues. The waived ethics approval was approved by the Ethics Committee of Xiangya Hospital of Central South University.

\section{Consent for Publication}

Consent.

\section{Author Contributions}

All authors made a significant contribution to the work reported, whether that is in the conception, study design, execution, acquisition of data, analysis and interpretation, or in all these areas; took part in drafting, revising or critically reviewing the article; gave final approval of the version to be published; have agreed on the journal to which the article has been submitted; and agree to be accountable for all aspects of the work.

\section{Funding}

This study is supported by grants from the China Postdoctoral Science Foundation (2021T140754, 2020M672521), the Science and Technology Innovation Program of Hunan Province (2021RC3029), the Natural Science Foundation of Hunan Province (2020JJ5934), and the Postdoctoral Science Foundation of Central South University (248485).

\section{Disclosure}

The authors declare that they have no competing interests.

\section{References}

1. Fiste O, Liontos M, Koutsoukos K, Terpos E, Dimopoulos MA, Zagouri F. Circulating tumor DNA-based predictive biomarkers in breast cancer clinical trials: a narrative review. Ann Transl Med. 2020;8(23):1603. doi:10.21037/atm-20-1175

2. Hill HE, Schiemann WP, Varadan V. Understanding breast cancer disparities-A multi-scale challenge. Ann Transl Med. 2020;8(14):906. doi:10.21037/atm.2020.04.37

3. Jazieh K, Bell R, Agarwal N, Abraham J. Novel targeted therapies for metastatic breast cancer. Ann Transl Med. 2020;8(14):907. doi:10.21037/atm.2020.03.43

4. Xu Z, Peng B, Cai Y, et al. N6-methyladenosine RNA modification in cancer therapeutic resistance: current status and perspectives. Biochem Pharmacol. 2020;182:114258. doi:10.1016/j. bcp. 2020.114258

5. Yan Y, Liang Q, Xu Z, Yi Q. Integrative bioinformatics and experimental analysis revealed down-regulated CDC42EP3 as a novel prognostic target for ovarian cancer and its roles in immune infiltration. PeerJ. 2021;9:e12171. doi:10.7717/peerj.12171

6. Xu Y, Liu J, Chen WJ, et al. Regulation of N6-methyladenosine in the differentiation of cancer stem cells and their fate. Front Cell Dev Biol. 2020;8:561703. doi:10.3389/fcell.2020.561703

7. Li Y, Ge YZ, Xu L, Xu Z, Dou Q, Jia R. The potential roles of RNA N6-methyladenosine in urological tumors. Front Cell Dev Biol. 2020;8:579919. doi:10.3389/fcell.2020.579919

8. Fazi F, Fatica A. Interplay between N (6)-methyladenosine (m(6)A) and non-coding RNAs in cell development and cancer. Front Cell Dev Biol. 2019;7:116. doi:10.3389/fcell.2019.00116

9. Liu $\mathrm{T}$, Wei $\mathrm{Q}$, Jin $\mathrm{J}$, et al. The $\mathrm{m} 6 \mathrm{~A}$ reader YTHDF1 promotes ovarian cancer progression via augmenting EIF3C translation. Nucleic Acids Res. 2020;48(7):3816-3831. doi:10.1093/nar/gkaa048

10. Han J, Wang JZ, Yang X, et al. METTL3 promote tumor proliferation of bladder cancer by accelerating pri-miR221/222 maturation in m6A-dependent manner. Mol Cancer. 2019;18(1):110. doi:10.1186/ s12943-019-1036-9

11. Müller S, Glaß M, Singh AK, et al. IGF2BP1 promotes SRF-dependent transcription in cancer in a m6A- and miRNA-dependent manner. Nucleic Acids Res. 2019;47(1):375-390. doi:10.1093/nar/gky1012

12. Hinshaw DC, Shevde LA. The tumor microenvironment innately modulates cancer progression. Cancer Res. 2019;79(18):4557-4566. doi:10.1158/0008-5472.Can-18-3962 
13. Zhang L, Zhang M, Xu J, et al. The role of the programmed cell death protein-1/programmed death-ligand 1 pathway, regulatory $\mathrm{T}$ cells and $\mathrm{T}$ helper 17 cells in tumor immunity: a narrative review. Ann Transl Med. 2020;8(22):1526. doi:10.21037/atm-20-6719

14. Schreiber RD, Old LJ, Smyth MJ. Cancer immunoediting: integrating immunity's roles in cancer suppression and promotion. Science. 2011;331(6024):1565-1570. doi:10.1126/science.1203486

15. Xu Z, Zeng S, Gong Z, Yan Y. Exosome-based immunotherapy: a promising approach for cancer treatment. Mol Cancer. 2020;19 (1):160. doi:10.1186/s12943-020-01278-3

16. Jia Y, Liu L, Shan B. Future of immune checkpoint inhibitors: focus on tumor immune microenvironment. Ann Transl Med. 2020;8 (17):1095. doi:10.21037/atm-20-3735

17. Liang H, Deng H, Liang W, et al. Perioperative chemoimmunotherapy in a patient with stage IIIB non-small cell lung cancer. Ann Transl Med. 2020;8(5):245. doi:10.21037/atm.2020.01.118

18. Barrett T, Wilhite SE, Ledoux P, et al. NCBI GEO: archive for functional genomics data sets-update. Nucleic Acids Res. 2013;41 (Database issue):D991-5. doi:10.1093/nar/gks1193

19. Luo X, Li H, Liang J, et al. RMVar: an updated database of functional variants involved in RNA modifications. Nucleic Acids Res. 2021;49(D1):D1405-d12. doi:10.1093/nar/gkaa811

20. Rhodes DR, Yu J, Shanker K, et al. ONCOMINE: a cancer microarray database and integrated data-mining platform. Neoplasia. 2004;6(1):1-6. doi:10.1016/s1476-5586(04)80047-2

21. Tang Z, Li C, Kang B, Gao G, Li C, Zhang Z. GEPIA: a web server for cancer and normal gene expression profiling and interactive analyses. Nucleic Acids Res. 2017;45(W1):W98-w102. doi:10.1093/ nar/gkx 247

22. Chandrashekar DS, Bashel B, Balasubramanya SAH, et al. UALCAN: a portal for facilitating tumor subgroup gene expression and survival analyses. Neoplasia. 2017;19(8):649-658. doi:10.1016/j. neo.2017.05.002

23. Á B, Győrffy B. TNMplot.com: a web tool for the comparison of gene expression in normal, tumor and metastatic tissues. Int $\mathrm{J} \mathrm{Mol}$ Sci. 2021;22(5). doi:10.3390/ijms22052622

24. Győrffy B. Survival analysis across the entire transcriptome identifies biomarkers with the highest prognostic power in breast cancer. Comput Struct Biotechnol J. 2021;19:4101-4109. doi:10.1016/j. csbj.2021.07.014

25. Amelio I, Gostev M, Knight RA, Willis AE, Melino G, Antonov AV. DRUGSURV: a resource for repositioning of approved and experimental drugs in oncology based on patient survival information. Cell Death Dis. 2014;5(2):e1051. doi:10.1038/cddis.2014.9

26. Vasaikar SV, Straub P, Wang J, Zhang B. LinkedOmics: analyzing multi-omics data within and across 32 cancer types. Nucleic Acids Res. 2018;46(D1):D956-d63. doi:10.1093/nar/gkx1090

27. Li T, Fan J, Wang B, et al. TIMER: a web server for comprehensive analysis of tumor-infiltrating immune cells. Cancer Res. 2017;77(21): e108-e10. doi:10.1158/0008-5472.Can-17-0307

28. $\mathrm{Ru} \mathrm{B}$, Wong $\mathrm{CN}$, Tong $\mathrm{Y}$, et al. TISIDB: an integrated repository portal for tumor-immune system interactions. Bioinformatics. 2019;35(20):4200-4202. doi:10.1093/bioinformatics/btz210
29. Robin X, Turck N, Hainard A, et al. pROC: an open-source package for $\mathrm{R}$ and $\mathrm{S}+$ to analyze and compare ROC curves. BMC Bioinform. 2011;12(1):77. doi:10.1186/1471-2105-12-77

30. Soysal SD, Tzankov A, Muenst SE. Role of the tumor microenvironment in breast cancer. Pathobiology. 2015;82(3-4):142-152. doi:10.1159/000430499

31. Yoshihara K, Shahmoradgoli M, Martínez E, et al. Inferring tumour purity and stromal and immune cell admixture from expression data. Nat Commun. 2013;4(1):2612. doi:10.1038/ncomms3612

32. He L, Li H, Wu A, Peng Y, Shu G, Yin G. Functions of N6-methyladenosine and its role in cancer. Mol Cancer. 2019;18 (1):176. doi:10.1186/s12943-019-1109-9

33. Shi Y, Zheng C, Jin Y, et al. Reduced expression of METTL3 promotes metastasis of triple-negative breast cancer by m6A methylation-mediated COL3A1 up-regulation. Front Oncol. 2020;10:1126. doi:10.3389/fonc.2020.01126

34. Zhang C, Samanta D, Lu H, et al. Hypoxia induces the breast cancer stem cell phenotype by HIF-dependent and ALKBH5-mediated $\mathrm{m}^{6}$ A-demethylation of NANOG mRNA. Proc Natl Acad Sci U S A. 2016;113(14):E2047-56. doi:10.1073/pnas.1602883113

35. Anita R, Paramasivam A, Priyadharsini JV, Chitra S. The m6A readers YTHDF1 and YTHDF3 aberrations associated with metastasis and predict poor prognosis in breast cancer patients. Am J Cancer Res. 2020;10(8):2546-2554.

36. Chung W, Eum HH, Lee HO, et al. Single-cell RNA-seq enables comprehensive tumour and immune cell profiling in primary breast cancer. Nat Commun. 2017;8(1):15081. doi:10.1038/ncomms15081

37. Xiao Y, Ma D, Zhao S, et al. Multi-omics profiling reveals distinct microenvironment characterization and suggests immune escape mechanisms of triple-negative breast cancer. Clin Cancer Res. 2019;25(16):5002-5014. doi:10.1158/1078-0432.Ccr-18-3524

38. Shani O, Vorobyov T, Monteran L, et al. Fibroblast-derived IL33 facilitates breast cancer metastasis by modifying the immune microenvironment and driving type 2 immunity. Cancer Res. 2020;80 (23):5317-5329. doi:10.1158/0008-5472.Can-20-2116

39. Wu SY, Fu T, Jiang YZ, Shao ZM. Natural killer cells in cancer biology and therapy. Mol Cancer. 2020;19(1):120. doi:10.1186/ s12943-020-01238-x

40. Chan IS, Knútsdóttir H, Ramakrishnan G, et al. Cancer cells educate natural killer cells to a metastasis-promoting cell state. J Cell Biol. 2020;219(9). doi:10.1083/jcb.202001134

41. Gou L, Yue GG, Puno PT, Lau CB. A review on the relationship of mast cells and macrophages in breast cancer - Can herbs or natural products facilitate their anti-tumor effects? Pharmacol Res. 2021;164:105321. doi:10.1016/j.phrs.2020.105321

42. Li S, Liu M, Do MH, et al. Cancer immunotherapy via targeted TGF$\beta$ signalling blockade in $\mathrm{T}(\mathrm{H})$ cells. Nature. 2020;587 (7832):121-125. doi:10.1038/s41586-020-2850-3

43. Wang L, Simons DL, Lu X, et al. Connecting blood and intratumoral $\mathrm{T}(\mathrm{reg})$ cell activity in predicting future relapse in breast cancer. Nat Immunol. 2019;20(9):1220-1230. doi:10.1038/s41590-019-0429-7
International Journal of General Medicine

\section{Publish your work in this journal}

The International Journal of General Medicine is an international, peer-reviewed open-access journal that focuses on general and internal medicine, pathogenesis, epidemiology, diagnosis, monitoring and treatment protocols. The journal is characterized by the rapid reporting of reviews, original research and clinical studies across all disease areas. The manuscript management system is completely online and includes a very quick and fair peer-review system, which is all easy to use. Visit http://www.dovepress.com/ testimonials.php to read real quotes from published authors. 